\title{
Competencia digital ciudadana: análisis de tendencias en el ámbito educativo
}

\section{(Digital Competence for Citizen: Analysis of Trends in Education)}

\author{
Diana Marín Suelves \\ Universitat de Valencia (España) \\ Nuria Cuevas Monzonís \\ Universidad Internacional de Valencia (España) \\ Vicente Gabarda Méndez \\ Universitat de Valencia (España)
}

DOI: https://doi.org/10.5944/ried.24.2.30006

\section{Cómo referenciar este artículo:}

Marín Suelves, D., Cuevas Monzonís, N., y Gabarda Méndez, V. (2021). Competencia digital ciudadana: Análisis de tendencias en el ámbito educativo. RIED. Revista Iberoamericana de Educación a Distancia, 24(2), pp. 329-349. https://doi.org/10.5944/ried.24.2.30006

\section{Resumen}

La competencia digital ciudadana se ha ido integrando en los procesos formativos de manera desigual, a pesar de la existencia de marcos internacionales que promueven su inclusión en los diferentes sistemas educativos. Este estudio trata de aproximarse al fenómeno desde una perspectiva bibliométrica y bibliográfica. Por un lado, se explora la producción científica en términos cuantitativos, a partir del análisis de los 87 artículos seleccionados de las bases de datos Scopus y Web of Science, analizando cuestiones como el número de autores o de citas. Por otro lado, se analiza el contenido de las 47 publicaciones disponibles en abierto, abordando cuestiones como la etapa educativa, los participantes o las áreas de trabajo. Los resultados confirman el aumento de la producción científica en el último año y la posición relevante que ocupa España, así como la predominancia de investigaciones centradas en estudiantes de Educación Superior y sobre el conjunto de áreas del Marco Digcomp. Las investigaciones confirman la variedad de herramientas y medios para el fomento de la competencia digital, así como la identificación de algunos condicionantes básicos para su desarrollo, como la etapa educativa, la formación docente o las características del contexto institucional, proporcionando algunas cuestiones fundamentales para seguir trabajando su integración en las diferentes etapas educativas. 
Palabras clave: competencia digital; ciudadanía; educación; bibliometría.

\begin{abstract}
Citizen digital competence has been integrating unevenly into training processes, despite the existence of international frameworks that promote its inclusion in the different education systems. This study tries to approach the phenomenon from a bibliometric and bibliographic perspective. On the one hand, scientific production is explored in quantitative terms, based on the analysis of the 87 articles selected from the Scopus and Web of Science databases, analysing issues such as the number of authors or citations. On the other hand, the content of the 47 publications available in open access is analysed, addressing issues such as educational stage, participants or the framework area considered. The results confirm the increase in scientific production in the last year and the relevant position held by Spain, as well as the predominance of research focused on Higher Education students and on the set of areas of the Digcomp Framework. The research confirms the variety of tools and means for the promotion of digital competence, as well as the identification of some basic conditioning factors for its development, such as the educational stage, teacher training or the characteristics of the institutional context, providing some fundamental issues to continue working on its integrating in the different educational stages.
\end{abstract}

Keywords: digital competence; citizenship; education; bibliometrics.

Desde hace unos años se ha tratado de identificar, por parte de diferentes organismos, cuáles son las competencias básicas que cualquier ciudadano debe desarrollar en el ámbito digital para poder desarrollarse de un modo adecuado en el contexto social, económico y cultural en que vivimos.

Una aproximación compartida a nivel internacional en esta línea es la que ofrece el Marco de Competencia Digital Ciudadana (DigComp) de la Unión Europea, a cargo de Carretero et al. (2017). Esta propuesta, compuesta por cinco áreas de desarrollo competencial y un total de 21 descriptores, establece cuáles son las principales destrezas que deben tener los ciudadanos en materia digital, sirviendo de referencia para el establecimiento, entre otros, de políticas educativas que permitan facilitar el desarrollo de estas competencias desde el ámbito educativo.

Se parte de la idea, no obstante, de que la competencia digital se ha venido integrando en los sistemas educativos de forma generalizada en los últimos años a partir de su identificación como competencia clave para el aprendizaje permanente (Comisión Europea, 2006). Sin embargo, el modo en que se integra en las diferentes etapas, así como las cuestiones específicas que se trabajan pueden variar de manera considerable.

Bajo esta perspectiva, el presente estudio presenta un análisis bibliométrico y de contenido acerca del abordaje específico de la vertiente ciudadana de la competencia digital en el ámbito educativo. De este modo, se analizará la producción científica 
relacionada con este tema ofreciendo, igualmente, información acerca de la productividad, la colaboración, el impacto y la difusión. En relación al contenido, se pondrá el foco específicamente sobre algunas categorías específicas como la etapa educativa en que se centran los estudios, el número de participantes, el área competencial específica que se trabaja o los resultados que se derivan, tomando como referencia para ello el Marco Digcomp 2.1.

\section{LA COMPETENCIA DIGITAL CIUDADANA}

Los modos de comunicarnos y relacionarnos se han visto condicionados, en las últimas décadas, por la integración de las tecnologías en las diferentes esferas de desarrollo. De esta manera, los contextos profesionales, académicos y personales han cambiado, adaptándose a las nuevas circunstancias, que hacen preciso que los ciudadanos adquieran y desarrollen nuevas destrezas que les permitan interactuar en todas ellas de un modo eficaz.

No es de extrañar, bajo esta coyuntura, que las diferentes administraciones hayan dedicado esfuerzos a identificar una serie de competencias elementales para la competencia digital ciudadana, así como estrategias específicas para tratar de promoverlas. Una de las propuestas más importantes es la que ofrece la Unión Europea y que se materializa en el Marco de Competencia Digital para los Ciudadanos - DigComp, que cuenta con varias versiones. La primera de ellas, la versión 1.o. (2013) servía para realizar una primera aproximación a las áreas de competencia, que fueron actualizadas en la versión 2.o. (2016). Por su parte, la versión más reciente, la versión 2.1. (2017) aporta, además, algunos ejemplos para su aplicación en el ámbito educativo y de la empleabilidad.

El resultado de todo este proceso es un planteamiento de cinco áreas competenciales y 21 descriptores o competencias específicas vinculadas a las áreas (ver tabla 1). Además, establece 8 niveles de desarrollo para cada una de las 21 competencias, permitiendo identificar de este modo las necesidades específicas vinculadas a cada una de ellas.

\section{Tabla 1}

Áreas y competencias del Marco de Competencia Digital para los Ciudadanos (DigComp 2.1.)

\begin{tabular}{ll}
\multicolumn{1}{c}{$\begin{array}{c}\text { Áreas } \\
\text { competenciales }\end{array}$} & \multicolumn{1}{c}{ Competencias } \\
\hline $\begin{array}{l}\text { Información y } \\
\text { alfabetización } \\
\text { informacional }\end{array}$ & $\begin{array}{l}\text { Navegación, búsqueda y filtrado de información, datos y contenido } \\
\text { digital }\end{array}$ \\
$\begin{array}{l}\text { Evaluación de datos, información y contenido digital } \\
\text { Gestión de datos, información y contenido digital }\end{array}$ \\
\hline
\end{tabular}




\begin{tabular}{|c|c|}
\hline $\begin{array}{c}\text { Áreas } \\
\text { competenciales }\end{array}$ & Competencias \\
\hline $\begin{array}{l}\text { Comunicación y } \\
\text { colaboración }\end{array}$ & $\begin{array}{l}\text { Interacción mediante tecnologías digitales } \\
\text { Compartir mediante tecnologías digitales } \\
\text { Participación ciudadana en línea } \\
\text { Colaboración mediante tecnologías digitales } \\
\text { Netiqueta } \\
\text { Gestión de la identidad digital }\end{array}$ \\
\hline $\begin{array}{l}\text { Creación de } \\
\text { contenidos digitales }\end{array}$ & $\begin{array}{l}\text { Desarrollo de contenidos digitales } \\
\text { Integración y reelaboración de contenidos digitales } \\
\text { Derechos de autor y licencias } \\
\text { Programación }\end{array}$ \\
\hline Seguridad & $\begin{array}{l}\text { Protección de dispositivos } \\
\text { Protección de datos personales y privacidad } \\
\text { Protección de la salud y el bienestar } \\
\text { Protección del entorno }\end{array}$ \\
\hline $\begin{array}{l}\text { Resolución de } \\
\text { problemas }\end{array}$ & $\begin{array}{l}\text { Resolución de problemas técnicos } \\
\text { Identificación de necesidades y respuestas tecnológicas } \\
\text { Uso creativo de las tecnologías digitales } \\
\text { Identificación de lagunas en la competencia digital }\end{array}$ \\
\hline
\end{tabular}

Fuente: Elaboración propia

Podría considerarse, además, que estos planteamientos han adquirido una nueva dimensión en la situación derivada de la COVID-19, donde la utilización de las tecnologías ha aumentado (Consell del Audiovisual de Catalunya, 2020) tanto en el ámbito del ocio como en el académico. En este último, se ha generado la necesidad de diseñar e implementar nuevas modalidades formativas derivadas del cierre de los centros educativos (Zubillaga y Gortazar, 2020) y dando lugar a modelos híbridos que han tratado de trasladar a una modalidad en línea las actividades programadas inicialmente en un entorno presencial (García-Planas y Taberna, 2020). Esta situación ha puesto de manifiesto, entre otras cuestiones, que sigue existiendo una laguna importante por parte de los diferentes agentes de la comunidad educativa en materia de competencia digital (Alonso y Expósito, 2020; Murillo y Duk, 2020), y que esto puede repercutir en un diseño inadecuado de las acciones formativas que pueden generar desigualdades y controversias (Díez y Gajardo, 2020).

\section{LA INTEGRACIÓN DE LA COMPETENCIA DIGITAL CIUDADANA EN EL CURRÍCULUM: ANÁLISIS NORMATIVO}

Como se apuntaba anteriormente, la Comisión Europea (2006; 2018) identificó la competencia digital como una de las competencias clave para el aprendizaje permanente. Este hecho sirvió para poner de manifiesto la importancia de que todos 
los ciudadanos desarrollen destrezas específicas en materia digital, poniendo esta competencia a la misma altura que la competencia lingüística o la matemática.

Además, el reconocimiento de la competencia digital a nivel internacional sirvió igualmente para que las diferentes administraciones educativas comenzaran a implementar políticas para su integración curricular en las diferentes etapas, promoviendo su adquisición desde el ámbito educativo.

Por ejemplo, en el sistema educativo español, las primeras alusiones aparecen en la Ley Orgánica de Educación (2006), que fueron posteriormente materializadas en las normativas curriculares de Educación Infantil, Primaria, Secundaria y de Educación Superior, así como en la Ley Orgánica para la Mejora de la Calidad Educativa (2013) que siguen vigentes a día de hoy. Concretamente, esta última normativa contempla, desde el propio preámbulo, que las TIC son uno de los aspectos fundamentales para la reforma educativa y para el aprendizaje de los ciudadanos a lo largo de la vida, poniendo de manifiesto los beneficios para el diseño e implementación de los procesos de enseñanza y aprendizaje, así como su potencial para la individualización de estos procesos y la atención a la diversidad. En esta línea, y derivado del reconocimiento de la competencia digital como competencia clave, se plantea su integración de un modo transversal en el currículum, considerando que debe abordarse en el marco de las diferente materias y asignaturas. Este abordaje se concibe desde una óptica no sólo instrumental, sino también actitudinal, considerando que el sistema educativo debe promover el uso responsable y ordenado de las tecnologías. Es importante reseñar, en el contexto del presente trabajo, que se considera uno de los fines del sistema educativo la preparación para el ejercicio de la ciudadanía y para la participación activa en la vida económica, social y cultural, con actitud crítica y responsable y con capacidad de adaptación a las situaciones cambiantes propias de la sociedad del conocimiento, siendo implícita la necesidad de formar a los ciudadanos en aquellas cuestiones que definen la sociedad en que vivimos.

Este punto de partida legislativo evidencia un interés institucional por la promoción de la competencia digital ciudadana por parte de la administración educativa (al menos desde un punto de vista normativo) y pone de manifiesto algunas directrices básicas de su integración como el abordaje transversal, su consideración eminentemente instrumental y la escasa vinculación con el desarrollo de la ciudadanía. Sin embargo, este planteamiento normativo ha permitido la generación de múltiples experiencias en el contexto educativo nacional e internacional donde se ha tratado de promover la competencia digital de los estudiantes de las diferentes etapas. Ejemplo de ello son los trabajos de López et al. (2019a) centrado en Educación Infantil, el estudio de Casado y Checa (2020) desarrollado en Educación Primaria, la propuesta de López et al. (2019b) contextualizado en Educación Secundaria o la investigación de Colomo et al. (2020) desarrollado en Educación Superior.

El análisis de esta cuestión en el sistema educativo español servirá de punto de partida para el presente estudio, que tiene por objetivo analizar la producción científica relacionada con la promoción de la competencia digital ciudadana en la 
última década desde un punto de vista bibliométrico y de contenido, tratando de tener una visión más holística del relieve que tiene este fenómeno en los diferentes sistemas educativos.

\section{MÉTODO}

Este estudio descriptivo de carácter restrospectivo (Moreno, 2019) se ha realizado desde la perspectiva cienciométrica. Los estudios bibliométricos permiten mostrar un panorama general de un campo de estudio y las tendencias en investigación (Zulueta y Bordons, 1999) a través de la cuantificación de los rasgos fundamentales de la literatura científica, como en este caso la competencia digital en el contexto educativo para el desarrollo de la ciudadanía del siglo XXI.

Se utilizaron como bases de datos Scopus, por su reconocido prestigio (García et al., 2020) y WOS, para superar limitaciones de estudios previos (Abad-Segura et al., 2020; Campos et al., 2020).

La búsqueda se realizó el 1 de julio de 2020, para conocer la tendencia de este año. Los términos utilizados para la búsqueda fueron: digital competence OR digital skills AND education AND citizens OR society, en título, palabras clave y resumen. Se decidió no incluir términos relacionados de manera exclusiva con cada una de las diferentes áreas, bajo la idea de que la competencia digital ciudadana se vertebra de un modo integral. Se incluyeron todos los artículos excepto los de revisión y los criterios de exclusión fueron: repeticiones, teóricos, validaciones de instrumentos, no centrados en el objeto de estudio y otra tipología de documentos. Sin utilizar filtro temporal se seleccionaron un total de 87 para su análisis, publicados en la última década. En la figura 1 se muestra el proceso siguiendo el Método PRISMA (Urrútia y Bonfill, 2010). Los artículos fueron seleccionados con una elevada fiabilidad intersujetos y un grado de acuerdo muy bueno (Altman, 1991) con un resultado en la prueba kappa de Cohen de o,8849. 


\section{Figura 1}

Diagrama de flujo (método PRISMA)
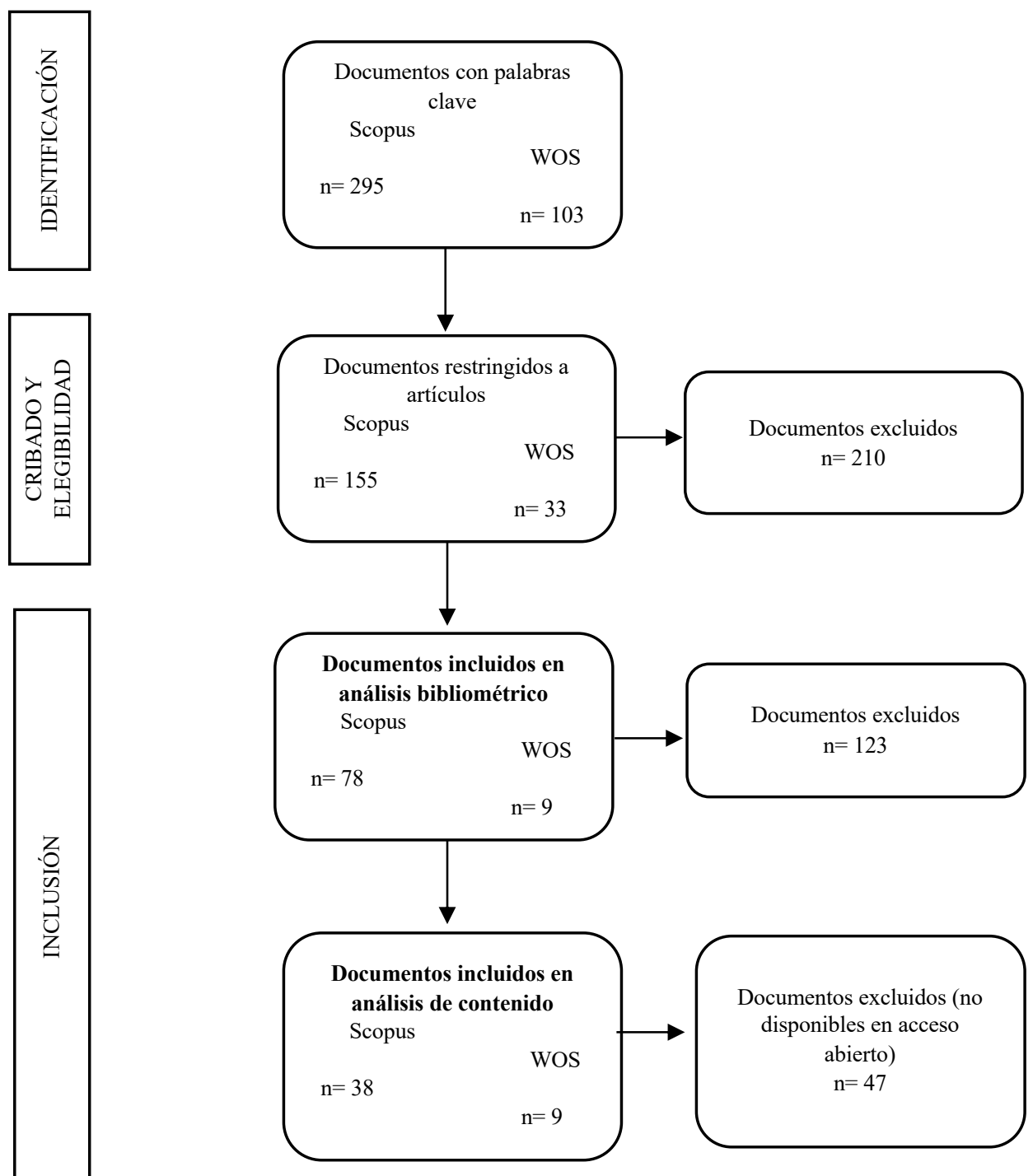

En segundo lugar, y para complementar la información obtenida en el estudio bibliométrico, se realizó un análisis de contenido, a partir de los 47 artículos disponibles en acceso abierto en ambas bases de datos. 
El análisis se realizó a partir de indicadores bibliométricos de productividad, colaboración, dispersión e impacto (Aleixandre, 2010) y el análisis de contenido a través de una ficha elaborada ad hoc en la que se describió la muestra, las etapas educativas, las áreas y los resultados obtenidos.

Para la representación gráfica de los resultados se utilizó la herramienta de software VOSviewer versión 1.6.11 (Van Eck y Waltman, 2011).

\section{RESULTADOS}

A fin de dar respuesta a los objetivos del estudio, se ofrecen a continuación los resultados organizados en dos bloques: el análisis bibliométrico y el análisis de contenido.

\section{Análisis bibliométrico}

En primer lugar, respecto a la productividad científica, se analizó la cantidad de documentos publicados, sin limitar a un área ni a un periodo concreto. En la figura 2 se puede observar una tendencia ascendente en la última década, y destacan los datos de 2020, dados los artículos publicados en el primer semestre.

\section{Figura 2}

Artículos por año (Scopus y Web of Science)

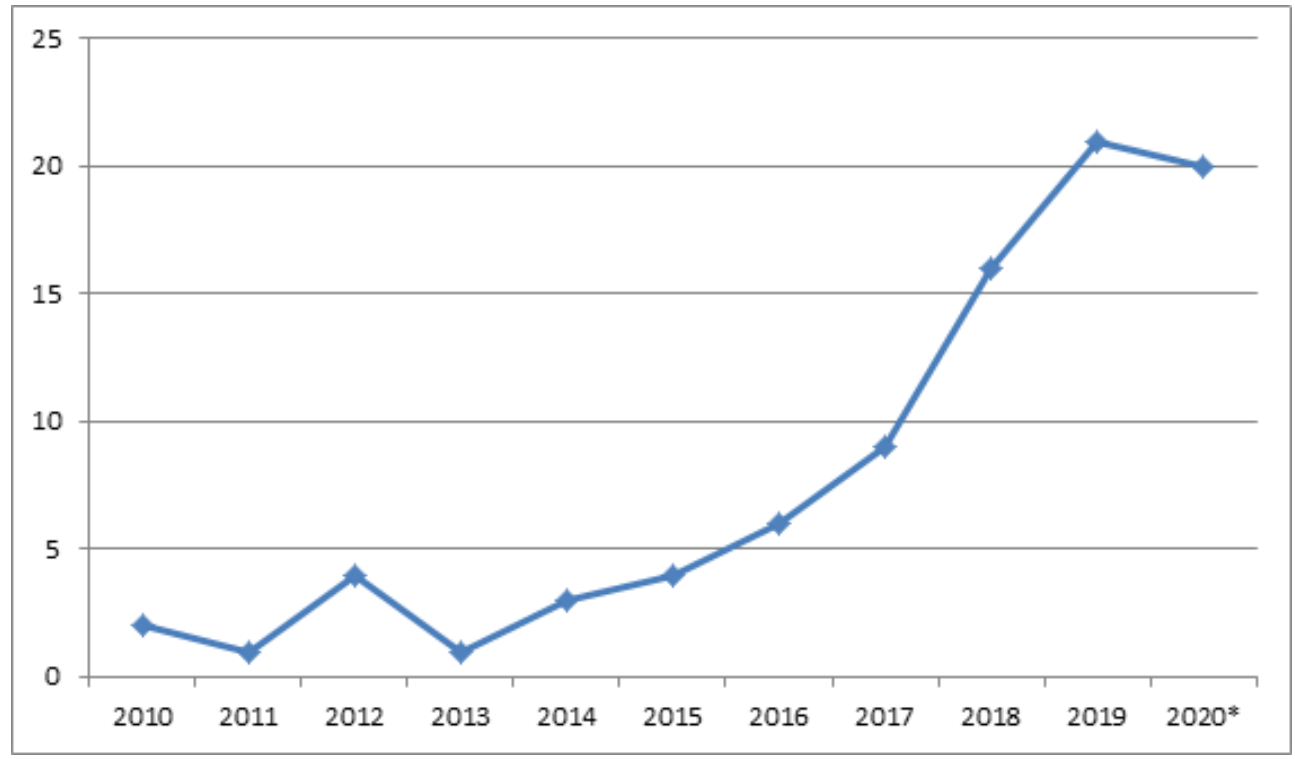


Con base en la ley de Lotka, se confirma que en este campo los productores son ocasionales, ya que, todos ellos cuentan con tan solo una publicación, excepto Gréta Börjk Gudmundóttir de la Universitetet i Oslo (Hardersen y Gudmundsdóttir, 2012; Hatlevik et al., 2015) y Rosa García-Ruiz de la Universidad de Cantabria (Amor et al., 2015; García-Ruiz et al., 2014; García-Ruiz et al., 2020) que han colaborado en dos y tres artículos respectivamente.

En cuanto al peso de cada país en el abordaje de la competencia digital ciudadana a través de la publicación de artículos científicos, es destacable el papel de España, que cuenta con casi la mitad de las publicaciones $(48,3 \%)$, seguida de países como EEUU (5,7\%), Italia (4,6\%) o Suecia (4,6\%). Pero, además, existen artículos escritos desde 32 países, pertenecientes a 4 continentes, tan distantes y diferentes como Colombia (Conde et al., 2017) e India (Nedungadi et al., 2018), lo que indica el amplio interés por el análisis de este tema a nivel mundial. A pesar del dato anterior, el idioma elegido para la difusión del conocimiento es el inglés en la mayoría de los artículos (65,5\%), seguido del español (28,7\%).

En segundo lugar, destaca la existencia de importantes redes de colaboración entre investigadores, ya que, son 247 autores los que firman los 87 artículos analizados. En la tabla 2 se muestra como la mayoría de los artículos fueron escritos por dos o más autores (82,8\%), siendo el artículo de Jara et al. (2015) el que cuenta con un mayor número de autores.

\section{Tabla 2}

Número de autores por artículo

\begin{tabular}{ccc}
\hline Número de autores & $\mathbf{N}$ & $\%$ \\
\hline 1 & 15 & 17,2 \\
\hline 2 & 16 & 18,4 \\
\hline 3 & 34 & 39,1 \\
\hline 4 & 10 & 11,5 \\
\hline 5 & 9 & 10,3 \\
\hline Más de 5 & 3 & 3,4 \\
\hline
\end{tabular}

Fuente: Elaboración propia

Analizando la filiación de los autores predominan las colaboraciones entre miembros de una misma institución $(51,4 \%)$ o de instituciones ubicadas en un mismo territorio (ciudad, Estado o país) que representan un 32\%, mientras que, un 13,9\% son artículos firmados por autores de diferentes países, como el de Ascencio et al. (2019), procedentes de Chile, México y España, o el firmado por Cook et al. (2018), que cuenta con representantes de EEUU, Canadá y Países Bajos.

En tercer lugar, la dispersión analizada mediante la ley de Bradford, relaciona el número de revistas y los artículos publicados sobre una temática. En este caso 
el núcleo está formado por un número de revistas reducido $(\mathrm{n}=10)$ en las que se han publicado un cuarto de los artículos analizados $(n=22)$. Mientras que no es posible diferenciar entre la zona 1 y 2 , ya que, el resto de artículos no se concentran en ninguna revista, sino que cada uno de los 65 artículos restantes aparecen en una revista diferente $(n=65)$. Las revistas en las que aparecen mayor cantidad de artículos centrados en la CDC son Profesorado y la Revista de Educación a Distancia, con tres artículos en cada una de ellas.

En cuarto y último lugar, en el estudio bibliométrico se analizó la visibilidad o impacto de las publicaciones, partiendo de las áreas de conocimiento desde las que se generan artículos científicos, las instituciones, las citas que recibe cada documento y la red de citación entre autores.

Por lo que respecta a las áreas de conocimiento desde las que se genera el conocimiento en torno a la CDC, es predominante el peso del área de las Ciencias Sociales $(48,7 \%)$, la Psicología (14,9\%) y la Informática (13,8\%), pero existen documentos generados desde otras áreas como la Ingeniería $(11,5 \%)$, las Artes y Humanidades $(4,6 \%)$ o las Ciencias de la Salud $(2,3 \%)$.

En este campo firman los artículos investigadores pertenecientes a instituciones de Educación Superior entre las que destacan las universidades españolas de Salamanca, Granada o Valencia, y con financiación de fundaciones, asociaciones o ministerios.

En cuanto al impacto medido por las citas recibidas, la tabla 3 evidencia un impacto nulo o escaso de los artículos, ya que, más de 3/4 de ellos han recibido un máximo de 10 citas, mientras que sólo dos artículos superan el centenar (Calvani et al., 2012; Gutiérrez y Tyner, 2012).

\section{Tabla 3}

Número de citas

\begin{tabular}{ccc}
\hline Número de citas & $\mathbf{N}$ & $\%$ \\
\hline o & 34 & 39,1 \\
\hline entre 1 y 10 & 37 & 42,5 \\
\hline entre 11 y 20 & 11 & 12,6 \\
\hline entre 21 y 30 & 1 & 1,1 \\
\hline entre 31 y 40 & 2 & 2,2 \\
\hline 100 o más & 2 & 2,2 \\
\hline
\end{tabular}

Fuente: Elaboración propia

Para finalizar, en la figura 3 se muestra la red de citación entre autores apareciendo investigadores de reconocido prestigio en el estudio del impacto de las tecnologías en educación. 


\section{Figura 3}

Red de citación entre autores (Vosviewer)

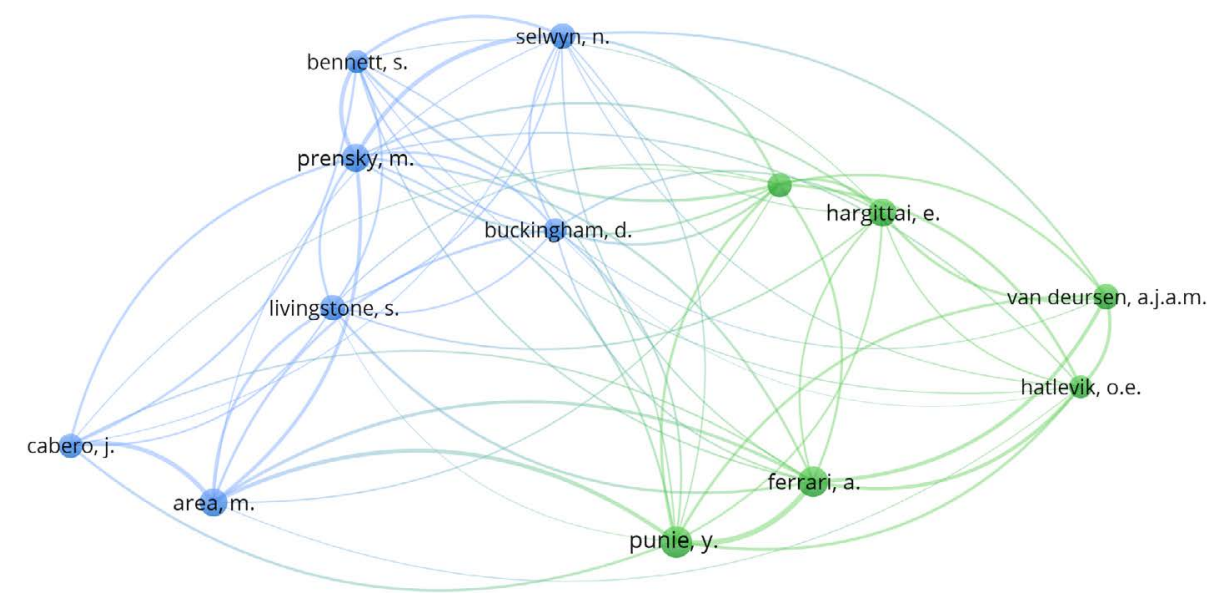

Vosviewer

\section{Análisis de contenido}

El análisis de contenido se realiza a través de la exploración de cuatro categorías: la etapa educativa en que se centra el estudio, el número de participantes que compone la muestra, las áreas del Marco Digcomp que se trabajan y los resultados que se derivan de los estudios. Se aborda, de este modo, el análisis de la producción científica de un modo más holístico, tratando de dar respuesta a la limitación de la bibliometría, centrada tradicionalmente en el cómputo de publicaciones (AleixandreBenavent et al., 2017).

Respecto a la primera categoría, centrada en las etapas educativas en las que se fomenta la competencia digital ciudadana, la mayor parte de los estudios se concentran en Educación Superior (Aguilar et al., 2020; Aleandri y Consoli, 2020; Ata y Yildirim, 2019; McGuiness y Fulton, 2019; Napal et al., 2018), siendo reseñable que se contextualizan casi todos ellos en la rama de Ciencias Sociales. Es igualmente destacable que, en los diversos estudios se contempla el abordaje de esta competencia de modos muy diversos. Desde el enfoque curricular, Napal et al. (2018) apuestan por un diseño transversal desde las diferentes áreas del marco Digcomp 2.1., mientras que Ata y Yildirim (2019), por ejemplo, realizan una formación específica para el profesorado en formación sobre el desarrollo de esta competencia. También son diversas las metodologías que se utilizan para ello, pudiendo observarse la autobiografía como recurso para el abordaje de este fenómeno (Aleandri y Consoli, 
2020) o su desarrollo mediante el aprendizaje de otros conceptos curriculares (Aguilar et al., 2020). También es reseñable la cantidad de artículos que se centran en la escolaridad obligatoria, especialmente en la etapa de Educación Primaria (Fraga-Varela et al., 2019; García-Ruiz et al., 2020; Lores et al., 2019; Pérez-Escoda et al., 2016) o que combinan los resultados de estas etapas con otras, como Infantil o Secundaria (Castro et al., 2019; Fajardo et al., 2016; Monteagudo-Fernández et al., 2020). En el caso de estas publicaciones, vinculadas a la escolaridad pre-obligatoria y postobligatoria, es subrayable también la variedad de enfoques y conceptos con que se vincula la competencia digital ciudadana: en algunos casos se vincula con cuestiones curriculares como la lectura (Fajardo et al. 2016), mientras que, en otros, se asocia en mayor medida con cuestiones vinculadas a la esfera más personal (Lores et al., 2019; Pérez-Escoda et al., 2016).

En relación a la segunda categoría, orientada al número de participantes en investigaciones, se encuentra algo de dispersión. De este modo, hay grandes muestras de participantes como las de García-Ruiz et al. (2020) o Gros et al. (2012), que superan el millar; otros estudios con muestras menos numerosas, aunque destacables como las de Basantes-Andrade et al. (2020), Llorent-Vaquero et al. (2020) o Amor et al. (2015); y otras muestras más pequeñas (menores de 100 participantes) como las que presentan Gregmigni (2018), Hervás-Torres (2020) o Shopova (2014).

Analizando, como tercera categoría, las áreas del Marco de Competencia Digital que se abordan en los estudios, lo más habitual es que se analicen cuestiones asociadas a las cinco áreas (1. Información y alfabetización informacional; 2) Comunicación y colaboración; 3) Creación de contenidos digitales; 4) Seguridad y 5) Resolución de problemas). Este es el caso, por ejemplo, de las propuestas de Bolek et al. (2018), Esteve-Mon et al. (2019) o Hinojo-Lucena et al. (2019). También resulta reseñable que, cuando las investigaciones se centran en una única área, suele ser en la de Información y alfabetización informacional, como las de Dolničar et al. (2020) y Valverde-Crespo et al. (2018) o de esta área con alguna otra, como en los casos de Marta-Lazo et al. (2019) y Maureen et al. (2020). En todo caso, es reseñable que, independientemente del área que se desee desarrollar, se proponen contextos de aprendizaje de carácter transversal, como un SMOOC (Marta-Lazo et al., 2019) y metodologías globales como el storytelling (Maureen et al., 2020) o la robótica (Esteve-Mon et al., 2019), no pudiendo inferirse una relación inequívoca entre el método y el área.

En último lugar, atendiendo a los resultados de las investigaciones y, más concretamente al abordaje de la competencia digital en ellas, se concluye que la integración de Tecnologías de la Información y la Comunicación en los procesos de enseñanza y aprendizaje contribuyen a una mejora de la competencia tanto en el contexto escolar inmediato (McGuiness y Fulton, 2019) como en el aprendizaje a lo largo de la vida (Aleandri y Consoli, 2020). Los niveles competenciales son mayores en las áreas de Información y alfabetización informacional (Ata y Yildirim, 2019; Shopova, 2014) y en la de Comunicación (Fraga-Varela et al., 2019; Llorent- 
Vaquero et al., 2020). Además, se concluye que el nivel de competencia digital está relacionado con la edad (Basantes-Andrade et al., 2020; Mirke et al., 2019) y el nivel educativo (Dolničar et al., 2020), así como de factores organizativos que están más allá de los roles docentes y discentes (Hinojo-Lucena et al., 2019; Sjöberg y Lilja, 2019). Por último, se concluye que los procesos formativos donde se contempla el abordaje de la competencia digital tiene un impacto positivo sobre el desarrollo de cuestiones como la atención a la diversidad (Castro et al., 2019), la comprensión de los contenidos (Aguilar et al., 2020), el empoderamiento y participación ciudadana (Cabero-Almenara et al., 2019), el pensamiento computacional (Esteve-Mon et al., 2019), el compromiso profesional (Hervás-Torres, 2020), el trabajo colaborativo (Aleandri y Consoli, 2020) o la motivación (Maureen et al., 2020), consideradas todas ellas destrezas básicas para el desarrollo de una ciudadanía efectiva en la sociedad en que vivimos.

Para finalizar el análisis de contenido, la figura 4 presenta un mapa de coocurrencia, con los términos más habituales de los estudios analizados. Asimismo, el mapa recoge la tendencia de los conceptos a aparecer juntos, permitiendo identificar tres clusters principales de contenido. El primero de ellos, en azul, articula los conceptos claves que giran en torno a los dos ejes de nuestro estudio (educación y destrezas digitales), relacionándose con una gran cantidad de términos como ciudadanía, ciudadanía digital, internet, brecha digital, enseñanza o alfabetización mediática. Por otro lado, el cluster en color amarillo ayuda a trasladar estos conceptos a los procesos formativos, girando en torno al término "competencia digital" y asociándose a conceptos como Educación Primaria o Educación Secundaria. Por último, el cluster de color verde completa nuestro mapa, identificando a los estudiantes como principal agente en los estudios analizados, así como la Educación Superior, etapa donde se concentran la mayor parte de ellos y la alfabetización digital, objetivo básico de los estudios. 


\section{Figura 4}

Mapa de co-ocurrencia (VosViewer)

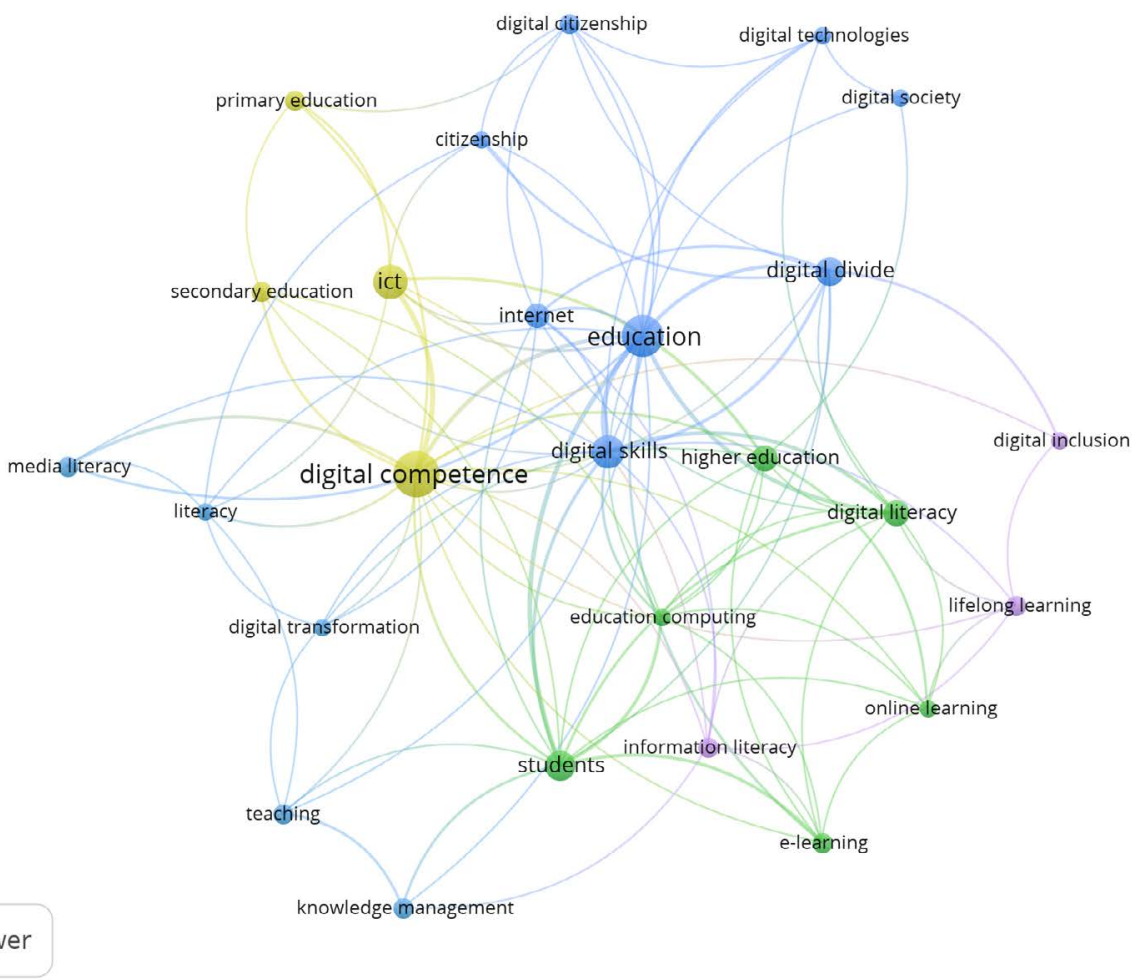

Estos datos ayudan a entender que, en la situación actual derivada del COVID-19, el alcance de los estudios sobre competencia digital ciudadana ha de concebirse desde una perspectiva amplia, teniendo impacto en las diferentes etapas educativas y sobre elementos diversos (personales e instrumentales) que conforman los escenarios formativos.

\section{DISCUSIÓN Y CONCLUSIONES}

La producción científica relacionada con la competencia digital ciudadana ha sido un fenómeno en auge en los últimos años. Es lógico si se tiene en cuenta que hasta finales de la década pasada no cristalizó el concepto y, durante la presente, se han diseñado e implementado estudios y experiencias para fomentar su desarrollo en los diferentes contextos. 
Atendiendo a los resultados de este estudio bibliométrico, frente a otros estudios anteriores relacionados con las tecnologías y la educación (Abad-Segura et al., 2020), destacan de manera relevante que gran parte de ella se concentra en España, dando sentido al reconocimiento de la competencia digital como competencia clave y las directrices establecidas en sus normativas para su integración curricular. Sin embargo, los resultados coinciden con estos otros en que el idioma predominante es el inglés, poniendo de manifiesto el interés de los investigadores por difundir sus estudios a nivel internacional, y en que suelen ser, mayoritariamente, publicaciones que se realizan en coautoría (dos o tres autores de la misma institución). El peso de las áreas de Ciencias Sociales y Psicología, como en el trabajo de Marín et al. (2020), evidencia la importancia del desarrollo de esta competencia en el ámbito educativo. Asimismo, es especialmente reseñable la producción científica del último año, 2020, que puede guardar relación con la necesaria implementación de formación híbrida o en línea asociada a la situación derivada de la COVID-19 en diferentes etapas educativas.

En relación al análisis de contenido, puede verse una integración creciente de la competencia digital ciudadana en las diferentes etapas, aunque está más presente en educación superior. Aun así, el enfoque con que se aborda en las diferentes etapas parece tener ciertas similitudes, siendo habitual que se trabaje de un modo transversal y mediante herramientas y metodologías de carácter diverso. Este hecho, sin embargo, debería contraponerse con la literatura científica acerca de la competencia digital de los docentes, considerando que la destreza de este colectivo tiene un impacto directo tanto en la calidad de los diseños formativos como en los resultados que finalmente puedan inferirse de cada uno de los estudios.

Por último, en cuanto a las limitaciones de este estudio se encuentra el acceso restringido a algunos documentos y la reciente publicación de la mayor parte de los artículos lo que explica el escaso impacto obtenido, siendo este un aspecto que resultaría interesante analizar pasado un tiempo, en combinación con el análisis de las altmétricas (Sixto et al., 2019). Asimismo, convendría incluir en el análisis otras bases de datos reconocidas que permitieran dotar al estudio de un carácter más global, así como utilizar de manera específica las diferentes áreas del marco Digcomp 2.1. como términos de búsqueda para partir del análisis realizado desde una perspectiva integral en este trabajo a un estudio con base en las áreas que componen la competencia digital.

\section{REFERENCIAS}

Abad-Segura, E., González-Zamar, M. D., de la Rosa, A. L., y Gallardo-Pérez, J. (2020). Gestión de la economía digital en la educación superior: tendencias y perspectivas futuras. Campus Virtuales, 9(1), 57-68.
Aguilar, I., Alejo, V. A., y Ayala, J. (2020). Desarrollo de objetos de aprendizaje para el aprendizaje de las estructuras de datos. Innoeduca. International Journal of Technology and Educational Innovation, 
6(1), 42-55. https://doi.org/10.24310/ innoeduca.2020.v6i1.5297

Aleandri, G., y Consoli, E. (2020). Autobiographical methods and coding for increasing self-awareness and transversal skills. Journal of Educational, Cultural and Psychological Studies, 21, 275-300. https://doi.org/10.7358/ecps-2020-021alea

Aleixandre, R. (2010). Bibliometría e indicadores de producción científica. En J. Jiménez, J. M. Argimon, A. Martín y T. Vilardell (Eds.), Publicación científica biomédica: cómo escribir y publicar un artículo de investigación, 363-384. Editorial Elsevier. https:// doi.org/10.1016/B978-84-8086-4619.50027-8

Aleixandre-Benavent, R., González, J., Castelló, L., Navarro, C., Alonso-Arroyo, A., Vidal-Infer, A., y Lucas-Domínguez, R. (2017). Bibliometría e indicadores de actividad científica (II). Indicadores de producción científica en pediatría. Acta Pediátrica Española, 75(3-4), 44-50.

Alonso, F. M., y Expósito, J. (2020). De una escuela presencial a la virtual en 24 horas, ¿es posible? Aula de Innovación Educativa, 293, 22-26.

Altman, D. (1991). Practical statistics for medical research. CRC Press. https://doi. org/10.1201/9780429258589

Amor, M., Pérez-García, A., y García-Ruiz, R. (2015). La competencia mediática en educación infantil. Análisis del nivel de desarrollo en España. Universitas Psychologica, 14(2), 619-630. https://doi. org/10.11144/Javeriana.upsy14-2.cmei

Ascencio, P., Glasserman, L. G., y Quintana, J. (2019). Digital competences: Reality of students starting university life. Digital Education Review, 36, 68-84.

Ata, R., y Yildirim, K. (2019). Turkish preservice teachers' perceptions of digital citizenship in education programs. Journal of Information Technology
Education, 18, 419-436. https://doi. org/10.28945/4392

Basantes-Andrade, A., Cabezas-González, M., y Casillas-Martín, S. (2020). Digital competences relationship between gender and generation of university professors. International Journal of Advanced Science, Engineering and Information Technology, 1, 205-2011. https://doi. org/10.18517/ijaseit.10.1.10806

Bolek, V., Kokles, M., Romanová, A., y Zelina, M. (2018). Information literacy of managers: Models and factors. Journal of Business Economics and Management, 19(5), 722-741. https://doi.org/10.3846/ jbem.2018.6906

Cabero-Almenara, J., Torres-Barzabal, L., y Hermosilla-Rodríguez, J.M. (2019). Las TIC y la creación de una ciudadanía crítica e-digital. Education in the Knowledge Society, 2O, 1-10. https://doi. org/10.14201/eks2019 $20 \quad$ a22

Calvani, A., Fini, A., Ranieri, M., y Picci, P. (2012). Are young generations in secondary school digitally competent? A study on italian teenagers. Computers and Education, 58(2), 797-807. https:// doi.org/10.1016/j.compedu.2011.10.004

Campos, M. N., Navas-Parejo, M. R., y Moreno, A. J. (2020). Realidad virtual y motivación en el contexto educativo: Estudio bibliométrico de los últimos veinte años de Scopus. ALTERIDAD. Revista de Educación, 15(1), 47-60. https://doi. org/10.17163/alt.v15n1.2020.04

Carretero, S., Vuorikari, R., y Punie, Y. (2017). DigComp 2.1: The Digital Competence Framework for Citizens with eight proficiency levels and examples of use. http://doi.org/10.2760/38842

Casado, R., y Checa, M. (2020). Robótica y Proyectos STEAM: Desarrollo de la creatividad en las aulas de Educación Primaria. Píxel-Bit. Revista de Medios y Educación, 58, 51-69. https://doi. org/10.12795/pixelbit.73672 
Castro, M., Marín, D., y Saiz, H. (2019). Competencia digital e inclusión educativa. Visiones de profesorado, alumnado y familias. Revista de Educación a Distancia, 19(61). https://doi. org $/ 10.6018 / \mathrm{red} / 61 / 06$

Colomo, E., Gabarda, V., Cívico, A., y Cuevas, N. (2020). Percepción de estudiantes sobre el uso del videoblog como recurso digital en educación superior. Píxel-Bit Revista de Medios y Educación, Preprint. https://doi.org/10.12795/pixelbit.74358

Comisión Europea (2006). Recomendación 2006/962/CE del Parlamento Europeo y del Consejo, de 18 de diciembre de 2006, sobre las competencias clave para el aprendizaje permanente. Diario Oficial de la Unión Europea de 30.12.2006. http://eur-lex.europa.eu/legal-content/ $\mathrm{ES} / \mathrm{TXT} /$ ?uri=celex\%3A32006Hog62

Consejo Europeo (2018). Recomendación del Consejo de 22 de mayo de 2018 relativa a las competencias clave para el aprendizaje permanente. Diario Oficial de la Unión Europea de 04.06.2018. https://eurlex.europa.eu/legal-content/ES/TXT/ PDF/?uri=CELEX:32018Ho604(01)\&from $=\mathrm{SV}$

Conde, E., Trujillo, J. J., y Castaño, H. (2017). Deciphering the curriculum through ICT: An interactive vision on the digital competences of students of sports science and physical activity. Revista de Humanidades, 31, 195-214.

Consell del Audiovisual de Cataluña (2020). Boletín de información sobre el audiovisual en Cataluña (BIAC). Núm. extra. Especial coronavirus 2. Barcelona: CAC, Mayo de 2020. https://www.cac. cat/sites/default/files/2020-05/BIAC Coronavirus2 ES.pdf

Cook, K., Cakirlar, C., Goddard, T., Demuth, R. C., y Wells, J. (2018). Teaching open science: Published data and digital literacy in archaeology classrooms. Advances in Archaeological Practice, 6(2), 144-156. https://doi.org/10.1017/aap.2018.5
Díez, E. J., y Gajardo, K. (2020). Educar y evaluar en tiempos de Coronavirus: la situación en España. Multidisciplinary Journal of Educational Research, 10(2), 102-134. $\quad$ https://doi.org/10.17583/ remie.2020.5604

Dolničar, D., Podgornik, B.B., Bartol, T., y Šorgo, A. (2020). Added value of secondary school education toward development of information literacy of adolescents. Library \& Information Science Research, 42(2). https://doi. org/10.1016/j.lisr.2020.101016

Esteve-Mon, F. M., Adell-Segura, J., Nebot, M. A., Novella, G. V., y Aparicio, J. P. (2019). The development of computational thinking in student teachers through an intervention with educational robotics. Journal of Information Technology Education: Innovations in Practice, 18, 139-152. https://doi.org/10.28945/4442

Fajardo, I., Vilalta, E., y Salmerón, L. (2016). ¿Son realmente tan buenos los nativos digitales? Relación entre las habilidades digitales y la lectura digital. Anales de Psicología, 32(1), 89-97. https://doi. org/10.6018/analesps.32.1.185571

Fraga-Varela, F., Vila-Couñago, E., y Pernas-Morado, E. (2019). Aprendizajes ausentes en la Competencia Digital de preeadolescentes: Un estudio de casos pertenecientes a contextos socioculturales desfavorables. Revista de Educación a Distancia, 19(61). https://doi. org $/ 10.6018 / \mathrm{red} / 61 / 04$

García, I., Gallardo-López, J. A., y LópezNoguero, F. (2020). La inteligencia emocional y la educación emocional en la escuela: un estado de la cuestión a través del análisis bibliométrico de la producción científica en Scopus (2015-2019). En J. A. Marín, G. Gómez, M. Ramos y M. N. Campos (Eds.), Inclusión, tecnología y sociedad. Dyckinson S. L. (pp. 220-231).

García-Planas, M. I., y Taberna, J. (2020). The transition from the classroom to non-classroom teaching at the UPC 
during the COVID-19 pandemic. IJERI: International Journal of Educational Research and Innovation, (15), 177-187. https://doi.org/10.46661/ijeri.5015

García-Ruiz, R., Da Mota, A. P., ArenasFernández, A., y Ugalde, C. (2020). Alfabetización mediática en Educación Primaria. Perspectiva internacional del nivel de competencia mediática. PíxelBit. Revista de Medios y Educación, 58, 217-236. https://doi.org/10.12795/ pixelbit. 74535

García-Ruiz, R., Ramírez-García, A., y Rodríguez-Rosell, M. (2014). Media literacy education for a new prosumer citizenship. Comunicar, 43, 15-23. https://doi.org/10.3916/C43-2014-01

Gobierno de España (2006). Ley Orgánica 2/2006, de 3 de mayo, de Educación. Boletín oficial del Estado, 106(4), 1715817207.

Gobierno de España (2013). Ley Orgánica 8/2013, de 9 de diciembre, para la mejora de la calidad educativa. Boletín Oficial del Estado. https://www.boe.es/diario boe/ txt.php

Gremigni, E. (2018). Overcoming new forms of digital divide: Some remarks on the need for media education. Italian Sociological Review, 8(1), 81-102. http:// doi.org/10.13136/isr.v8i1.221

Gros, B., García, I., y Escofet, A. (2012). Beyond the net generation debate: A comparison of digital learners in face-toface and virtual universities. International Review of Research in Open and Distance Learning, 13(4), 190-2010. https://doi. org/10.19173/irrodl.v13i4.1305

Gutiérrez, A., y Tyner, K. (2012). Educación para los medios, alfabetización mediática y competencia digital. Comunicar, 19(38), 31-39. https://doi.org/10.3916/C382012-02-03

Hardersen, B., y Gudmundsdóttir, G. B. (2012). The digital universe of young children. Nordic Journal of Digital Literacy, 3, 221-226.
Hatlevik, O. E., Gudmundsdóttir, G. B., y Loi, M. (2015). Examining factors predicting students' digital competence. Journal of Information Technology Education, 14(1), 123-137. https://doi. org/10.28945/2126

Hervás-Torres, M. (2020). El entorno wiki y su aplicación didáctica innovadora. Revista de Educación a Distancia, 20(62). https://doi.org/10.6018/red.404161

Hinojo-Lucena, F. J., Aznar-Díaz, I., Cáceres-Reche, M. P., Trujillo-Torres, J. M., y Romero-Rodríguez, J. M. (2019). Factors Influencing the Development of Digital Competence in Teachers: Analysis of the Teaching Staff of Permanent Education Centres. IEEE Access, 7, 178744-178752. https://doi.org/10.1109/ ACCESS.2019.2957438

Jara, I., Claro, M., Hinostroza, J. E., San Martín, E., Rodríguez, P., Cabello, T., Ibieta, A., y Labbé, C. (2015). Understanding factors related to chilean students' digital skills: A mixed methods analysis. Computers and Education, 88, 387-398. https://doi.org/10.1016/j. compedu.2015.07.016

Llorent-Vaquero, M., Tallón-Rosales, S., y Monasterio, B. H. (2020). Use of information and communication technologies (ICTs) in communication and collaboration: A comparative study between university students from Spain and Italy. Sustainability, 12(10). https:// doi.org/10.3390/su12103969

López, J., Pozo, S., y López, G. (2019a). La eficacia de la Realidad Aumentada en las aulas de Infantil: un estudio del aprendizaje de SVB y RCP en discentes de 5 años. Píxel-Bit. Revista de Medios y Educación, 55, 157-178. https://doi. org/10.12795/pixelbit.2019.i55.09

López, M., Garro, E., y Egaña, T. (2019b). La lectura digital en un aula de secundaria: prácticas reales $\mathrm{y}$ dificultades del alumnado. Píxel-Bit. Revista de Medios 
y Educación, 55, 99-106. https://doi. org/10.12795/pixelbit.2019.i55.06

Lores, B., Sánchez, S., y García, R. (2019). La formación de la Competencia Digital en los Docentes. Profesorado. Revista de currículum y formación del profesorado, 23(4), 234-26o. https://doi. org/10.30827/profesorado.v23i4.11720

Marín, D., Castro, M. M., Peirats, J., y Rodríguez, J. (2020). Investigación bibliométrica en aprendizaje mediado por tecnología en alumnado de Altas Capacidades. Revista Brasileira de Educacao Especial, 26(2), 229246. https://doi.org/10.1590/1980$54702020 v 26 \mathrm{eo0} 76$

Marta-Lazo, C., Frau-Meigs, D., y OsunaAlcedo, S. (2019). A collaborative digital pedagogy experience in the tMOOC "Step by Step". Australasian Journal of Educational Technology, 35(5), 111-127. https://doi.org/10.14742/ajet.4215

Maureen, I. Y., Van der Meij, H., y De Jong, T. (2020). Enhancing Storytelling Activities to Support Early (Digital) Literacy Development in Early Childhood Education. International Journal of Early Chilhood, 52(1), 55-76. https://doi. org/10.1007/s13158-020-00263-7

McGuiness, C., y Fulton, C. (2019). Digital literacy in higher education: A case of study of student engagement with e-tutorials using blended learning. Journal of Information Technology Education: Innovations in Practice, 18, 1-28. https://doi.org/10.28945/4190

Mirke, E., Kasparová, E., y Cakula, S. (2019). Adults' readiness for online learning in the Czech Republic and Latvia (digital competence as a result of ICT education policy and information society development strategy. Periodicals of Engineering and Natural Sciences, 7(1), 205-215. https://doi.org/10.21533/pen. v7i1.366

Monteagudo-Fernández, J., RodríguezPérez, R. A., y Escribano-Miralles, A.
(2020). Percepciones de los estudiantes de Educación Secundaria sobre la enseñanza de la historia, a través del uso de las TIC y recursos digitales. Revista Electrónica Interuniversitaria de Formación del Profesorado, 23(2), 67-79. https://doi. org/10.6018/reifop.417611

Moreno, A. J. (2019). Estudio bibliométrico de la Producción Científica sobre la Inspección Educativa. REICE. Revista Iberoamericana sobre Calidad, Eficacia y Cambio en Educación, 17(3), 23-40. $\quad$ https://doi.org/10.15366/ reice2019.17.3.002

Murillo, F. J., y Duk, C. (2020). El Covid-19 y las Brechas Educativas. Revista latinoamericana de educación inclusiva, 14(1), 11-13. https://doi.org/10.4067/ S0718-73782020000100011

Napal, N., Peñalva-Vélez, A., y Mendióroz, A. (2018). Development of digital competence in secondary education teacher's training. Education Sciences, 8(3), 104-112. https://doi.org/10.3390/ educsci8030104

Nedungadi, P. P., Menon, R., Gutjahr, G., Erickson, L., y Raman, R. (2018). Towards an inclusive digital literacy framework for digital India. Education and Training, 6o(6), 516-528. https://doi.org/10.1108/ ET-03-2018-0061

Pérez-Escoda, A., Castro-Zubizarreta, A., y Fandos-Igado, M. (2016). Digital skills in the $\mathrm{Z}$ generation: Key questions for a curricular introduction in primary school. Comunicar, 24(49), 71-79. https://doi. org/10.3916/C49-2016-07

Shopova, T. (2014). Digital literacy of students and its improvement at the university. Journal on Efficiency and Responsibility in Education and Science, 7(2), 26-32. https://doi.org/10.7160/ eriesj.2014.070201

Sixto, A., Alonso, A., Lucas, R., González, J., y Aleixandre, R. (2019). Bibliometría e indicadores de actividad científica (XIV): Métricas alternativas o altmétricas. 
Nuevas formas de medir el impacto de la ciencia. Acta Pediátrica Española, 77(34), 44-52.

Sjöberg, J., y Lilja, P. (2019). University teachers' ambivalence about the digital transformation of higher education. International Journal of Learning, Teaching and Educational Research, 18(13), 133-149. https://doi. org/10.26803/ijlter.18.13.7

Urrútia, G., y Bonfill, X. (2010). Declaración PRISMA: una propuesta para mejorar la publicación de revisiones sistemáticas y metaanálisis. Medicina clínica, 135(11), 507-511. https://doi.org/10.1016/j. medcli.2010.01.015

Valverde-Crespo, D., De Pro-Bueno, A., y González-Sánchez, J. (2018). La competencia informacional-digital en la enseñanza y aprendizaje de las ciencias en la educación secundaria obligatoria actual: Una revisión teórica. Revista Eureka, 15(2), 1-15. https://doi.org/10.25267/ Rev Eureka ensen divulg cienc.2018. v15.i2.2105

Van Eck, N. J., y Waltman, L. (2011). Text mining and visualization using VOSviewer. ISSI Newsletter, 7(3), 50-54.

Zubillaga, A., y Gortazar, L. (2020). COVID-19 y educación: Problemas, respuestas y escenarios. Fundación Cotec para la Innovación. https://bit. ly/3auXnP8

Zulueta, M. A., y Bordons, M. (1999). A global approach to the study of teams in multidisciplinary research areas through bibliometric indicators. Research Evaluation, 8(2), 111-118. https://doi. org/10.3152/147154499781777612

\section{PERFIL ACADÉMICO Y PROFESIONAL DE LOS AUTORES}

Diana Marín Suelves. Las principales líneas de investigación en las que ha centrado su interés han sido la intervención psicoeducativa, la atención a la diversidad, la inclusión, el desarrollo positivo de niños y adolescentes y, como miembro del Grupo de investigación CRIE (GIUV2013-105), la competencia digital docente, las estrategias didácticas digitales y los procesos de digitalización de los contenidos curriculares. https://orcid.org/0000-0002-5346-8665

E-mail: diana.marin@uv.es

\section{DIRECCIÓN DE LA AUTORA}

Facultad de Filosofía y Ciencias de la Educación

Avda. Blasco Ibáñez, 30

46010

Valencia (España)

Nuria Cuevas Monzonís. Su trayectoria investigadora se ha centrado principalmente en dos líneas. Por un lado, trabaja sobre la familia como agente educativo, siendo miembro del Grupo de Investigación e Intervención en Escuela Violencia y Familia de la Universidad Internacional de Valencia, donde trabaja actualmente como Profesora Adjunta al Área de Educación. Por otro lado, la competencia digital docente constituye otra de sus líneas de trabajo fundamentales, 
especialmente en la formación inicial y la implementación de metodologías alternativas. https://orcid.org/0000-0001-9366-3038

E-mail: nuria.cuevas@campusviu.es

DIRECCIÓN DE LA AUTORA

C/ Pintor Sorolla, 21

46002

Valencia (España)

Vicente Gabarda Méndez. Sus principales líneas de investigación se han centrado en la formación del profesorado y la innovación educativa. Aborda ambas cuestiones desde los dos Grupos de Investigación a los que pertenece: Grupo de investigación CRIE (GIUV2013-105) de la Universitat de València y el Grupo de Investigación en Innovación y Tecnología Educativa de la Universidad de Málaga (SEJ-533) de la Universidad de Málaga. https://orcid.org/o0oo-0001-6159-5173 E-mail: vicente.gabarda@uv.es

DIRECCIÓN DEL AUTOR

Facultad de Filosofía y Ciencias de la Educación Avda. Blasco Ibáñez, 30

46010

Valencia (España)

Fecha de recepción del artículo: 15/02/2021

Fecha de aceptación del artículo: 08/03/2021

Fecha de aprobación para maquetación: 29/03/2021 